\title{
On the mesh dependence of non-linear mechanical finite element analysis
}

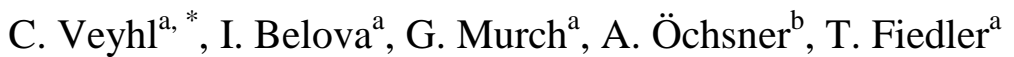

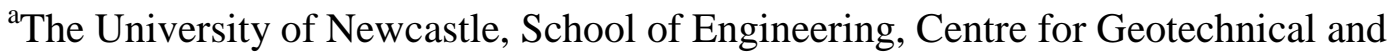
Materials Modelling, Centre for Mass and Thermal Transport in Engineering Materials, Callaghan, NSW 2308, Australia

${ }^{\mathrm{b}}$ Technical University of Malaysia, Department of Applied Mechanics, 81310 Skudai, Malaysia

*Corresponding author. Tel.: +61 249216198

E-mail address: christoph.veyhl@studentmail.newcastle.edu.au (C. Veyhl)

\begin{abstract}
This paper addresses the mesh-dependence of non-linear mechanical finite element analysis. To this end, finite element meshes that are assembled by various element types and their solutions are compared. Voxel, tetrahedron, hexahedron and mixed (hexdominant) meshes are considered. Different benchmarking parameters for the elastic and plastic solutions as well as for the computational load are determined. First, bending beams with a square, a circular and a rail cross-section are calculated accounting for non-linear material behavior (plasticity). A strong dependence on the mesh type is observed and the best results are obtained for mixed meshes and hexahedron-only meshes. In the second part of this study, finite element models that are based on the complex geometry of a metallic foam are considered. Computed tomography data is used to generate geometrically complex finite element models and a convergence analysis is performed. Again, superior performance is found for mixed meshes.
\end{abstract}

Keywords: Finite element method, plasticity, beam, mesh comparison, mixed (hexdominant) mesh, hexahedral mesh.

\section{Introduction}

Modern engineering materials such as cellular metals [1] exhibit complex geometries. Prediction of their mechanical properties by means of numerical finite element analysis requires an accurate representation of their very complex meso-structure $[2,3]$. To this end, computed micro-tomography $(\mu-\mathrm{CT})$ images can provide fairly accurate geometric information. However, the 3-D mesh generation is a complex task and the manual mesh generation becomes extremely time-consuming. Therefore an automatic, robust, accurate and repeatable meshing procedure is required. The main objective of the present research is to evaluate finite element mesh types for elasto-plastic material behaviour. This investigation is based on simple beam geometries and on a complex geometry obtained by computed tomography record. 
In the literature, two different strategies for mesh evaluation can be found. For selected geometries, analytical reference solutions exist and can be used for the verification of numerical results. However, more complex geometries and loading conditions often have no analytical solution and only the convergence of the numerical models can be compared. Wang et al. [4] simulated the bending of a beam with a square cross-section, fixed at one end and loaded on the other end. Only elastic material behaviour was considered. The analytical reference solution for the vertical displacement at the free end of the beam includes both a bending and a shear component. It was found that results of the use of quadratic tetrahedral and quadratic hexahedral elements are close to the analytical solution and that the linear tetrahedral element yields less accurate approximations. Cifuentes et al. [5] used the same geometry, material model and analytical solution as in the study before. They observed that quadratic tetrahedral and linear hexahedral elements are equivalent in terms of accuracy and processing time. As already observed in [4], quadratic tetrahedral elements yield better approximations than linear tetrahedral elements. Benzley et al. [6] extended the previous studies on a square beam by incorporating non-linear material behaviour (plasticity). Again, an analytical solution was used for the comparison with the numerical models. In the initial elastic range, the findings in $[4,5]$ could be confirmed, i.e. linear tetrahedral elements yield less accurate approximations. For non-linear material behaviour, it was found that both linear- and quadratic tetrahedral elements yield poor results. In contrast, linear- and quadratic hexahedrons provide more accurate results.

A few studies have been published that compared the convergence behaviour of different mesh types for complex geometries. Viceconti et al. [7] addressed a simplified model of a proximal femur bone and compared it with an analytical reference solution. Only elastic material behaviour was considered. Additionally, a more realistic model of a proximal femur bone was considered. No analytical solution was available for this complex geometry and therefore a convergence analysis was performed. In good agreement with the other approaches [4-6] it was found that hexahedral elements possess the most accurate results for the simplified geometric model and proximal femur bone. In addition, voxel meshes were considered. It was found that this method requires a large number of elements to reach the accuracy comparable to that of the other meshing approaches. Ramos et al. [8] addressed the same geometries (simplifiedand realistic proximal femur bone) as in the previous study by Viceconti [7]. In contradiction to the other studies, they observed that the simplified model of a proximal femur bone with linear tetrahedral elements is closer to the approximated analytical solution than the model with quadratic tetrahedrons, linear- and quadratic hexahedrons. The results obtained simulating the realistic proximal femur with first- and second order tetrahedral- and hexahedral elements did not show significant mesh dependence.

The present paper is based on a similar concept but takes the previous research further with important development. Beams with a square cross section, a circular cross section 
and a rail profile are investigated. The bending of a beam, fixed at one end and loaded at the other end is simulated and non-linear material behaviour is taken into account. Results of the square- and circular cross section beams are compared to an analytical reference solution. For the rail profile, an approximate analytical solution is obtained and also a convergence analysis is performed. In addition to the bending beams, a metallic foam with the complex geometry obtained by computed micro tomography is considered. No analytical solution is available for this complex geometry and therefore only a convergence analysis can be performed. This complex model was necessary to test the automatic mesh generation under more realistic conditions.

\section{Finite Element Analysis}

In the present study, finite element analyses are conducted in order to compare threedimensional solid element types. Full integration is used in all cases [9]. Linear- and quadratic hexahedral elements, linear- and quadratic tetrahedral elements, voxel meshes and mixed meshes (containing linear hexahedral-, linear tetrahedral- and pentahedral elements) are considered. The element type used in voxel meshes is identical to the one applied in the linear hexahedral meshes. The difference is that voxel meshes are assembled by uniform cubes whereas in a hexahedron mesh also distorted elements of different size may be used to achieve a more accurate geometric discretisation. The linear hexahedron element (MSC.Marc element type 7 [9]) is an eight-node, isoparametric, arbitrary element. This element uses trilinear interpolation functions and, accordingly, the strains are constant throughout the element. The stiffness of this element is formed using eight-point Gaussian integration. The quadratic hexahedron element (MSC.Marc element type 21 [9]) is a 20-node, isoparametric, arbitrary hexahedron. This element uses triquadratic interpolation functions to represent the displacements. The stiffness of this element is formed using 27-point Gaussian integration. The linear tetrahedron element (MSC.Marc element type 134 [9]) is a fournode, isoparametric three-dimensional element. This element uses linear interpolation functions and the strains are constant throughout the element. For those elements the numerical integration is done by using one point at the centroid of the element. The quadratic 10-node tetrahedron element (MSC.Marc element 127 [9]) is a second-order isoparametric three-dimensional element. The stiffness of this element is formed using four-point integration. The mixed mesh contains the linear tetrahedron and hexahedron elements mentioned above. In addition, 6-node pentahedral elements (MSC.Marc element 136 [9]) may be used in the mixed mesh. This element uses trilinear interpolation functions and consequently the strains are constant throughout the element. The stiffness of this element is formed using six-point Gaussian integration.

As discussed above, linear or higher order (i.e. quadratic) shape functions can be used in combination with the elements. However, the maximum number of elements is restricted by the available computer memory. Elements with higher-order shape functions (i.e. degrees of freedom) require additional memory to handle additional 
nodes and therefore fewer elements can be used resulting in a less accurate discretisation of complex geometries. Accordingly, predominantly linear shape functions are considered in the present study.

Two commercial software packages are used for automatic mesh generation. The first one is the commercial grid generation package Harpoon (Sharc Ltd). Harpoon is able to generate mixed, only-tetrahedrons and voxel meshes. For the mixed technique, Harpoon uses an octree technique [10] to mesh geometry using user-defined element size constraints. Mixed meshes are created in two steps. First, the volume is roughly approximated by a voxel type hexahedron mesh. In the second step, nodes are mapped on the surface and the surface is smoothed by adding additional tetrahedral- and pentahedral elements. The second software package HEXPRESS ${ }^{\mathrm{TM}}$ allows the generation of an only-hexahedron mesh. The input geometry is represented as a STL format surface. HEXPRESS ${ }^{\mathrm{TM}}$ requires a very clean and accurate representation of the surface prior to the generation of a volumetric mesh. In the first step, a rough mesh is created that encompasses the entire model. Then, the model is refined via an octree technique [11] so that cells are sufficiently small for capturing the details of the domain. At the next step, all cells of the octree-refined mesh that fall outside the domain or intersect its boundary are removed. The resulting mesh is then projected onto the surface geometry by moving nodes on the surface geometry. This method is also known as volume to surface approach. The commercial software Harpoon program has shown to be fast, robust and easy to use. In contrast, the meshing process with HEXPRESS ${ }^{\mathrm{TM}}$ is more time-intensive and at this time restricted to less complex geometries. The solution of the finite element models and the post-processing are realised with the commercial Software MSC.Marc (MSC Software Corp.).

The bending of a beam, fixed at one end and loaded on the other end is simulated (cf. Fig. 1a) and non-linear material behaviour (ideal-plasticity with no plastic hardening) is accounted for. Three-dimensional beam models with different cross sections (cf. Fig. 1b-d) are used for the assessment of different element types. The applied force on the end of the beam incrementally increases until the maximum force is reached. The numerical computation results are then compared to analytical solutions. The material properties of the beams are: Young's modulus $E=210000 \mathrm{~N} / \mathrm{mm}^{2}$, Poisson's ratio $v=$ 0.3 and initial yield stress $k=112.5 \mathrm{~N} / \mathrm{mm}^{2}$. 
a)

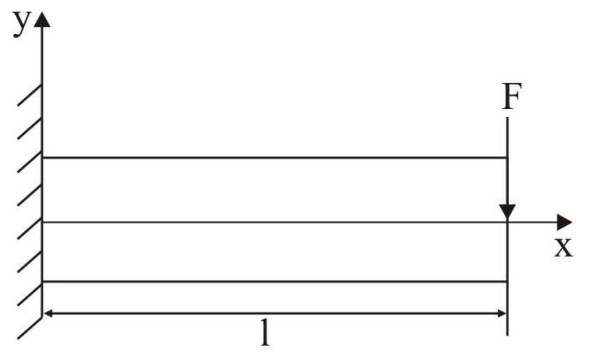

b)

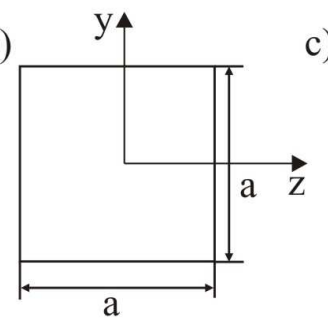

d)

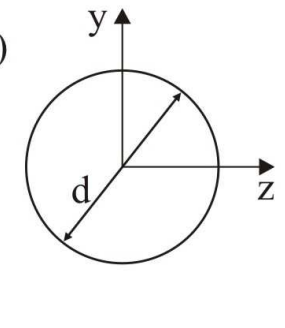

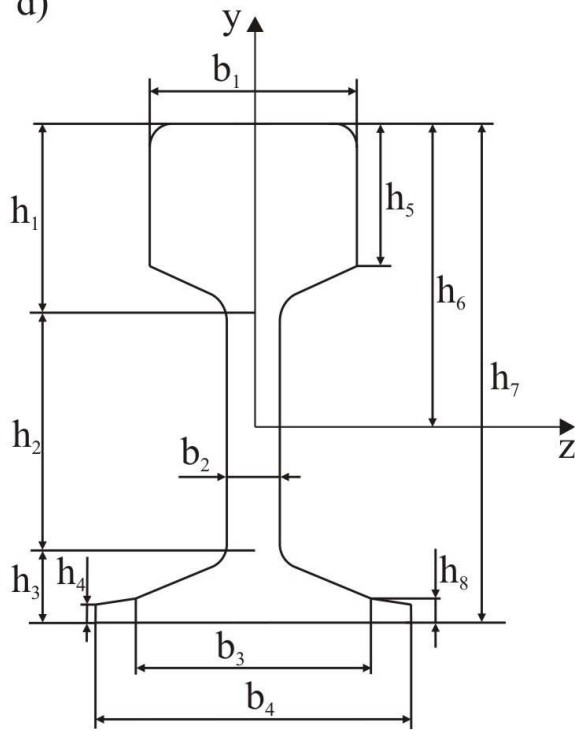

Fig. 1. Beam geometries: a) cantilever beam oriented in the $\mathrm{x}$-direction and loaded in the negative $y$-direction; b) square cross section $(l=100 \mathrm{~mm}, a=20 \mathrm{~mm})$; c) circular cross section $(l=100 \mathrm{~mm}, d=20 \mathrm{~mm})$; d) simplified rail profile $\mathrm{S} 54$ [15] $(l=473.11$ $\mathrm{mm}, b_{1}=70 \mathrm{~mm}, b_{2}=16 \mathrm{~mm}, b_{3}=87.7 \mathrm{~mm}, b_{4}=125 \mathrm{~mm}, h_{1}=55 \mathrm{~mm}, h_{2}=70 \mathrm{~mm}, h_{3}$ $\left.=29 \mathrm{~mm}, h_{4}=12 \mathrm{~mm}, h_{5}=43.3 \mathrm{~mm}, h_{6}=79 \mathrm{~mm}, h_{7}=154 \mathrm{~mm}, h_{8}=14.4 \mathrm{~mm}\right)$.

A second set of finite element models is based on computed tomography data of metallic foam (cf. Fig. 2a). Segmentation (identification of metallic and void phases) of the CT data was done using the imaging and editing software Mimics (Materialize). The obtained geometry was then meshed with the different meshing software packages mentioned earlier. Compressive loading was simulated according to the boundary conditions shown in Fig. 2b. The metallic foam is constrained at three surfaces (grey planes) by confining the displacement in the corresponding normal direction of each surface. Compressive loading is simulated by a time-dependent nodal displacement in the negative $y$-direction. A non-linear material model of $\mathrm{AlCu} 4 \mathrm{SiMg}$ (Aluminium 2014O) was applied [12] with $E=73100 \mathrm{~N} / \mathrm{mm}^{2}, v=0.33$ and $k=185 \mathrm{~N} / \mathrm{mm}^{2}$.

a)

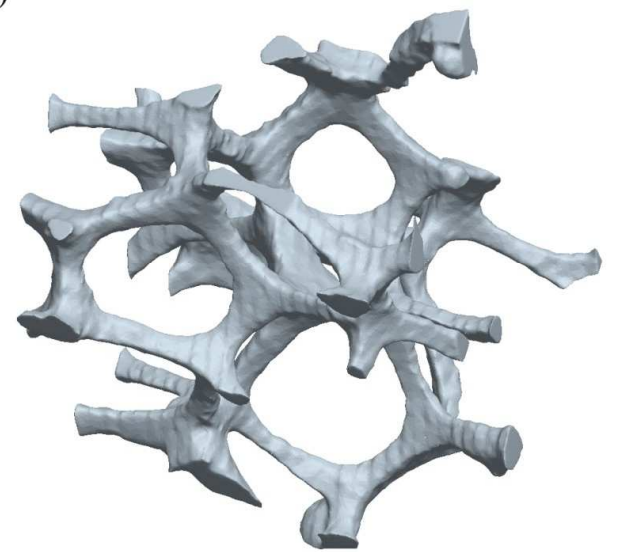

b)

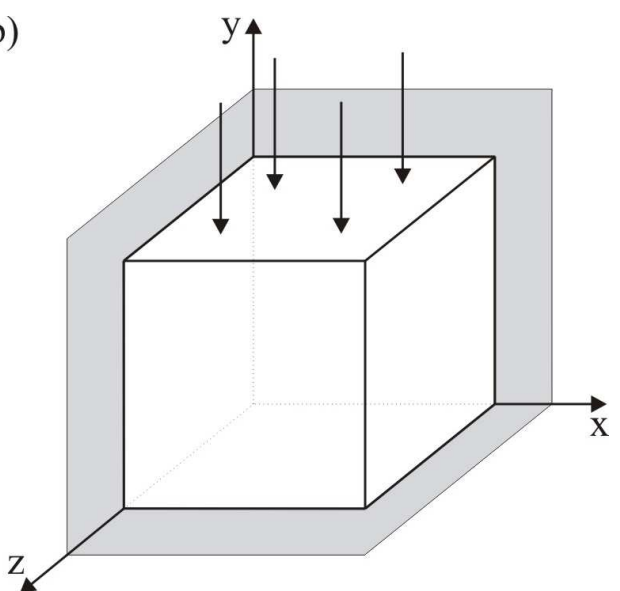

Fig. 2. Compressive testing: a) computed tomography 3D reconstruction of Empore ${ }^{\circledR}$ foam; b) schematic drawing of boundary conditions. 


\section{Analytical Solution}

The analytical expression for the vertical displacement of the centre-line at the free end of the beam is calculated for all beam profiles (cf. Fig. 1b-d). Elastic and elasto-plastic material behaviour is considered. The following equations for the elastic solution are taken from the engineering mechanic text [13]. The total deflection of the beam $w(x)$ is calculated with Eq. (1) and contains a bending $w_{\mathrm{b}}(x)$ and a shear $w_{\mathrm{s}}(x)$ component. For the geometries considered, bending is the dominant effect.

$$
w(x)=w_{\mathrm{b}}(x)+w_{\mathrm{s}}(x)
$$

The bending solution is calculated with Eq. (2) where $F$ is the applied Force, $l$ the total length of the beam, $E$ is Young's Modulus, $I_{z}$ the moment of inertia (see Table 1) and the $x$-coordinate is the distance from the fixed side:

$$
w_{\mathrm{b}}(x)=\frac{F \cdot l^{3}}{6 \cdot E \cdot I_{z}} \cdot\left(-\frac{x^{3}}{l^{3}}+3 \cdot \frac{x^{2}}{l^{2}}\right)
$$

The shear deflection $w_{\mathrm{s}}$ is calculated with Eq. (3) where $G$ is the shear modulus (for isotropic material behaviour see Eq. (4)) and the shear area $A_{\mathrm{s}}$ (see Table 1) which is the area $A$ of the cross section multiplied by the shear correction factor:

$$
\begin{aligned}
& w_{s}(x)=\frac{F}{G \cdot A_{s}} \cdot x \\
& G=\frac{E}{2 \cdot(1+v)}
\end{aligned}
$$

The analytical solution for plastic material behaviour is used as given in [14]. This solution is based on the main Bernoulli-Navier assumption that distribution of the stress component in the $x$ direction, $\sigma_{\mathrm{xx}}(=\sigma)$, is a function only of the $x$ and $y$ (see Fig. 1a) variables but not the $z$ variable. Moreover, only a linear distribution of the axial strain component $\varepsilon_{\mathrm{xx}}(=\varepsilon)$ is permitted in the elastic response regions.

\begin{tabular}{|l|l|l|l|}
\hline Cross Section & Square & Circle & $\begin{array}{l}\text { Rail (using data } \\
\text { given in Figure 1d) }\end{array}$ \\
\hline$I_{\mathrm{Z}}$ & $I_{z}=\frac{b_{1}^{4}}{12}$ & $I_{z}=\frac{\pi \cdot d^{4}}{64}$ & $\begin{array}{l}I_{z}=2.1348 \times 10^{7} \\
\mathrm{~mm}^{4}\end{array}$ \\
\hline$A_{\mathrm{S}}$ & $A_{s}=\frac{5}{6} \cdot b_{1}^{2}$ & $A_{s}=\frac{\pi \cdot d^{2}}{8}$ & $\begin{array}{l}A_{s}=0.42 A, \\
A=7031.81 \mathrm{~mm}^{2}\end{array}$ \\
\hline
\end{tabular}

Table 1: Equations for the moment of inertia and shear area for square-, circle- and rail cross sections.

The representative equivalent plastic strain distribution is shown in Figure 5a-d. It can be observed that these assumptions are only fulfilled in the case of the square and circular cross sections. The assumption is no longer valid in the case of the rail profile accordingly to the equivalent plastic strain distribution. In general, there is no restriction on the nature of irreversible inelastic response. However for the purposes of the present research we decided to use ideal-plasticity with no plastic hardening. 


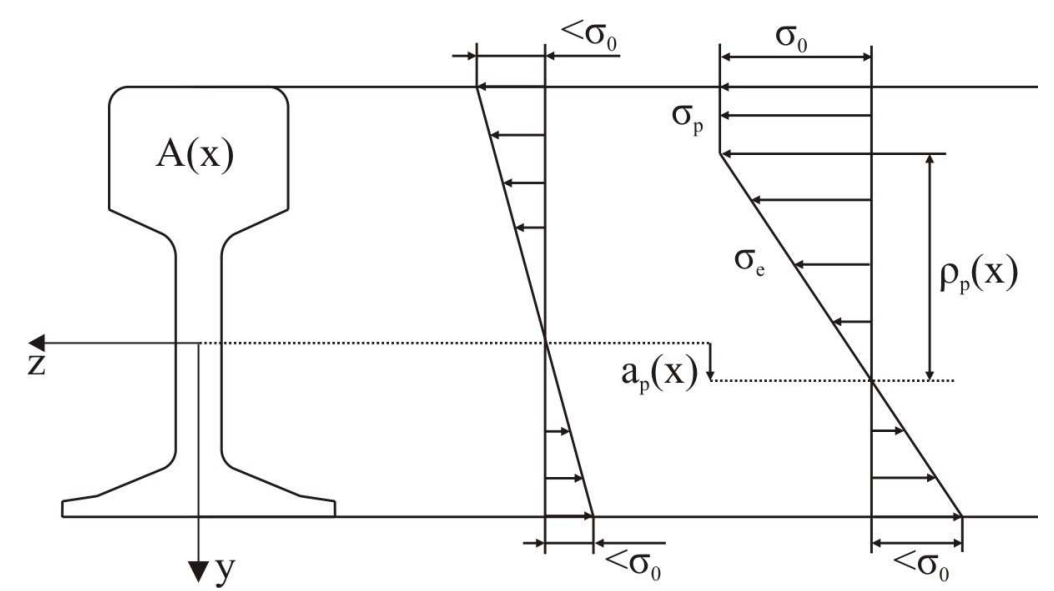

Fig. 3. Plastification and position of the neutral axis on a rail profile.

As a consequence of the main assumption the set of the quantities required for the problem to be solved is $\left\{a_{\mathrm{p}}(x), \rho_{\mathrm{p}}(x), w(x)\right\}$ where $a_{\mathrm{p}}$ and $\rho_{\mathrm{p}}$ describe the degree of plastification and its impact on the displacement of the neutral axis (cf. Fig. 3) and $w$ is the beam deflection in the y direction. Equations for $a_{\mathrm{p}}$ and $\rho_{\mathrm{p}}$ are:

$$
\begin{aligned}
& \int_{A_{p}(x)} \operatorname{sign}\left(y-a_{p}(x)\right) \sigma_{p} d A+\int_{A_{e}(x)} \sigma_{0}\left(\frac{y-a_{p}(x)}{\rho_{p}(x)}\right) d A=0 \\
& \int_{A_{p}(x)} \operatorname{sign}\left(y-a_{p}(x)\right) \sigma_{p} y d A+\int_{A_{e}(x)} \sigma_{0}\left(\frac{y-a_{p}(x)}{\rho_{p}(x)}\right) y d A=|M(x)|
\end{aligned}
$$

where $A(x)$ is the cross-section of the beam at $x ; A_{\mathrm{p}}(x)$ is the region of plastic deformations in $A(x): A_{\mathrm{p}}(x)=\left\{A(x) \cap\left\{(y, z), y>a_{\mathrm{p}}+\rho_{\mathrm{p}}\right\} \cap\left\{(y, z), y<a_{\mathrm{p}}-\rho_{\mathrm{p}}\right\}\right\}$; whereas $A_{\mathrm{e}}(x)$ is the region of elastic only deformations in $A(x): A_{\mathrm{e}}(x)=\left\{A(x)-A_{\mathrm{p}}(x)\right\}$. $A_{\mathrm{e}}(x)$ is located centrally around the actual neutral axis. $M(x)$ is the applied moment; $\sigma_{0}$ is the yield stress; $\sigma_{\mathrm{p}}$ is the plastic stress which in general can be a function of plastic deformations and the $y$ and $x$ variables, but in our chosen model for plasticity without plastic hardening we have that $\sigma_{\mathrm{p}}$ is constant and equal to $\sigma_{0}$.

Accordingly, the equation for $w(x)$ yield:

$$
\frac{d^{2} w(x)}{d x^{2}}= \begin{cases}-\frac{M(x)}{E I}, & x \in \Omega_{e} \\ \operatorname{sign}(-M(x)) \frac{\sigma_{0}}{E \rho_{p}(x)}, & x \in \Omega_{p},\end{cases}
$$

where $\Omega_{\mathrm{e}}$ and $\Omega_{\mathrm{p}}$ are elastic and plastic sections of the beam, respectively. These sections are made by cutting the beam perpendicular in the $x$ direction. In $\Omega_{\mathrm{e}}$ there is no plastic deformation (or yield stress is not exceeded) and $\Omega_{\mathrm{p}}$ is the remaining part of the beam. $\Omega_{\mathrm{p}}$ comprises all cross sections that are characterized by the presence of plastic deformations. Obviously, in $\Omega_{\mathrm{e}}$ the elastic solution outlined above is followed. 
If the load $F$ is lower than the critical value $F_{\text {cr }}=a^{3} k / 6 l$ then only linear deformations are present in the beam. Once the load $F$ is greater than the critical value $F_{\text {cr }}$ plastic deformations are developed in the beam.

For the elasto-plastic region $\Omega_{\mathrm{p}}$ solution for the square beam can be found in closed form as follows. For the functions $\rho_{\mathrm{p}}(x)$ and $w_{\mathrm{p}}(x)$ we have following expressions:

$$
\begin{aligned}
& \rho_{p}(x)=\frac{a}{2} \sqrt{3\left(1-\frac{4|M(x)|}{a^{3} k}\right)} \\
& w_{p}(x)=\frac{4 K}{3 m_{1}^{2}} \sqrt{\left(M_{p}-|M(x)|\right)^{3}}+c_{1} x+c_{0}
\end{aligned}
$$

where $M_{\mathrm{p}}=\sigma_{0} b_{1}{ }^{3} ; K=\operatorname{sign}(-M(x)) \sqrt{\frac{b_{1} \sigma_{0}^{3}}{3 E^{2}}} ;$ applied moment has the form $M(x)=F(-x$ $+l)=m_{1} x+m_{0}$; constants $c_{1}$ and $c_{0}$ can be found from the boundary conditions between elastic only (described by general Eq. (1-3) and elasto-plastic regions (Eq. (11)) where $w(x)$ and its first derivative must be continuous functions.

Now the volume $\mathrm{V}_{\mathrm{p}}$ of the plastic region in $\Omega_{\mathrm{p}}$ can be calculated as follows:

$$
V_{p}=2 \int_{0}^{x_{c r}} \int_{0}^{a} \int_{\rho_{p}(x)}^{a / 2} 1 d y d z d x=\frac{a^{2}}{18 F}\left(18 F l-4 a^{3} k+3\left(a^{3} k-4 F l\right) \sqrt{3-\frac{12 F l}{a^{3} k}}\right)
$$

For the cases of circular and rail beams for each load $F$ (and associated moment $M(x)$ ) we solved Eq. (9) for a set of points in the elasto-plastic region $\Omega_{\mathrm{p}}$ and then integrated Eq. (10) numerically (using the solution for the elastic region - Eq. (1-3)). The resulting values are presented in the next section.

\section{Results and Discussion}

\subsection{Bending beams}

Numerical computations for all beams are performed with the following mesh types: mixed, linear hexahedron, linear tetrahedron and voxel. Beam models with different numbers of nodes $(10000,50000$ and 150000$)$ are investigated and the elastic gradient, maximum deflection, plasticity volume and processing time are evaluated. Analytical force-deflection curves and selected numerical solutions with the least deviation are plotted in Figure 4. It can be observed that the analytical solution of the square- and circular beam is in excellent agreement with the corresponding numerical solutions (see Fig. 4a). In contrast, Figure 4b shows that the analytical solution of the rail profile deviates from the numerical solution. The reason for this deviation are the simplifications presumed for the analytical solution, i.e. that the plastic strain has a constant horizontal distribution in the $y z$-plane. The area where the equivalent plastic strain obtained by finite element analysis is not equal to zero is coloured grey in Figure 5. Figure 5d shows that the numerical computation of the rail profile leads to a curved plastic strain distribution that strongly deviates from the analytical assumption. In 
contrast, the square and circular beams (cf. Figure $5 \mathrm{~b}, \mathrm{c}$ ) show only a very minor deviation between the assumed analytical and the numerical plastic strain distribution. Accordingly, good agreement of the analytical and numerical solutions is obtained. Due to the poor agreement between numerical and analytical solution in case of the rail profile, a high-resolution finite element model (300 000 nodes) is used as reference instead.
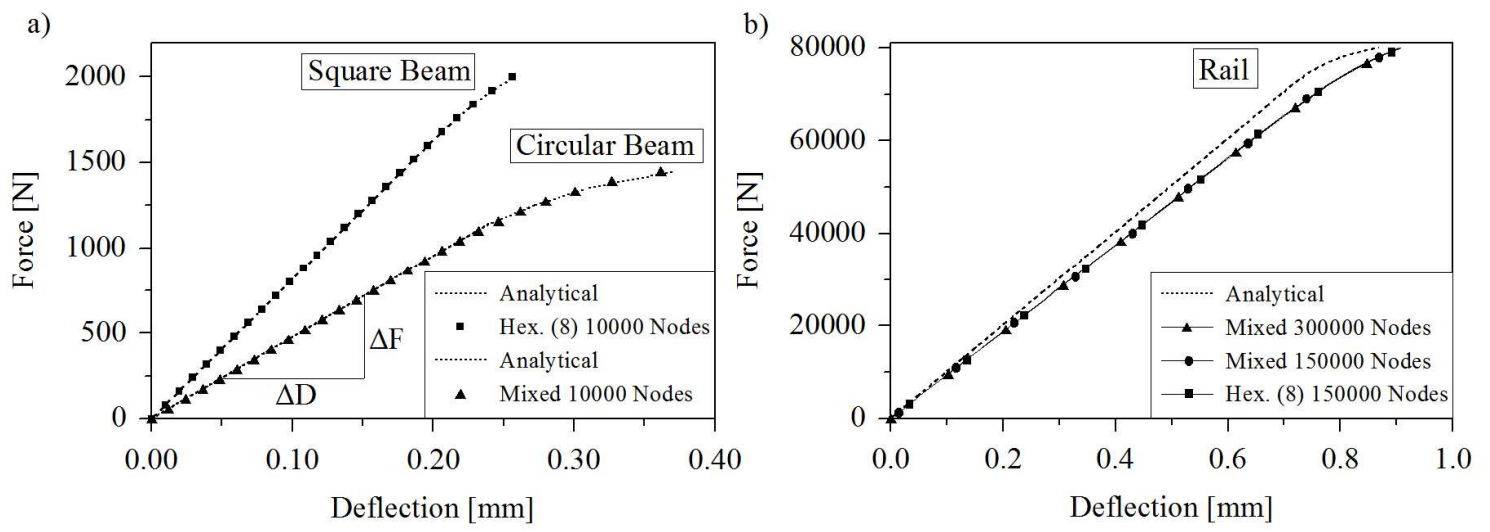

Fig. 4. Force-deflection-curves: a) square- and circular beam; b) rail profile.

a)

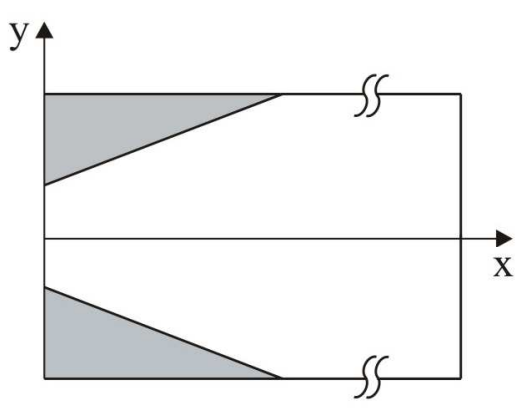

b)

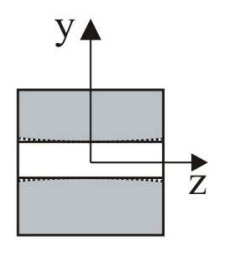

c)

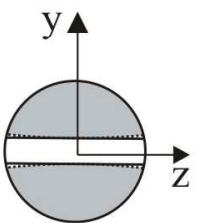

d)

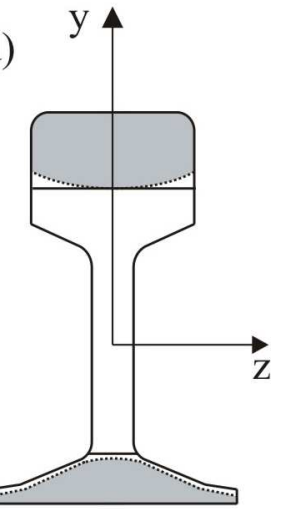

\section{Analytical Assumption ……..... Numerical Solution}

Fig. 5. Total equivalent plastic strain distribution: a) longitudinal view of the squareand circular beam; b) cross section of the square beam; c) cross section of the circular beam; d) cross section of the rail.

For the comparison of the different mesh types first the elastic gradient is considered. The elastic gradient is obtained from the initial constant slope $\Delta F / \Delta D$ of the forcedisplacement curve in the elastic region (cf. Fig. 4a). Figure 6a shows the deviation to the reference solution. As expected, an increase of the number of elements also decreases the deviation. It can be observed that the mixed meshes achieve the most accurate results independent of the cross-section. In good agreement with earlier results $[4,5,6]$, the highest deviation is observed for the linear tetrahedral elements. 
The maximum displacement at the end of the beam obtained for the maximum load $F$ is addressed in Figure 6b. It should be mentioned here that according to Eq. (1) this value contains elastic and a plastic component. The absolute deflection deviation quantifies the difference between the numerical and reference solutions. As already observed for the elastic solution the most accurate results are obtained by the mixed mesh. Likewise, the linear hexahedron meshes exhibit consistently good results and poor accuracy is found for the linear tetrahedrons.

The initial deformation of the beam is purely elastic. Plastification starts at higher bending loads at the upper and lower surface at the clamping where the maximum stresses arise. In the case of the symmetric square- and circular beams also the plastic deformation field develops symmetrically. For the rail profile, the plasticity starts at the upper surface due to higher stresses (cf. Fig. 3). The load is then increased incrementally and the volume of plastic deformation continues to grow and propagate. This volume is used for further benchmarking of different mesh types for non-linear material behaviour (cf. Fig. 6c). The reference values for the square beam (load $F=$ $2000 \mathrm{~N}$ ) and circular beam (load $F=1450 \mathrm{~N}$ ) are obtained by making use of the analytical solution. They are $1925 \mathrm{~mm}^{3}$ (direct use of the Eq. (13)) and $2885 \mathrm{~mm}^{3}$ (numerical integration of Eq. (10)), respectively. For the rail profile, the reference value $164245 \mathrm{~mm}^{3}$ is obtained by the finite element reference model (mixed mesh, 300000 nodes, load $F=80000 \mathrm{~N}$ ). It can be observed that in accordance with the previous results, mixed meshes achieve the most accurate results and linear tetrahedron meshes show the highest deviation.

The processing time (computational weight) is shown in Fig. 6d. The simulations were done on a DELL Precision T7400 machine with Windows Vista 64-Bit, $2.5 \mathrm{GHz}$ and 64 GByte of RAM memory. It can be seen that linear hexahedral elements require the longest calculation times. Surprisingly, small calculation times are observed for mixed meshes. 
a)

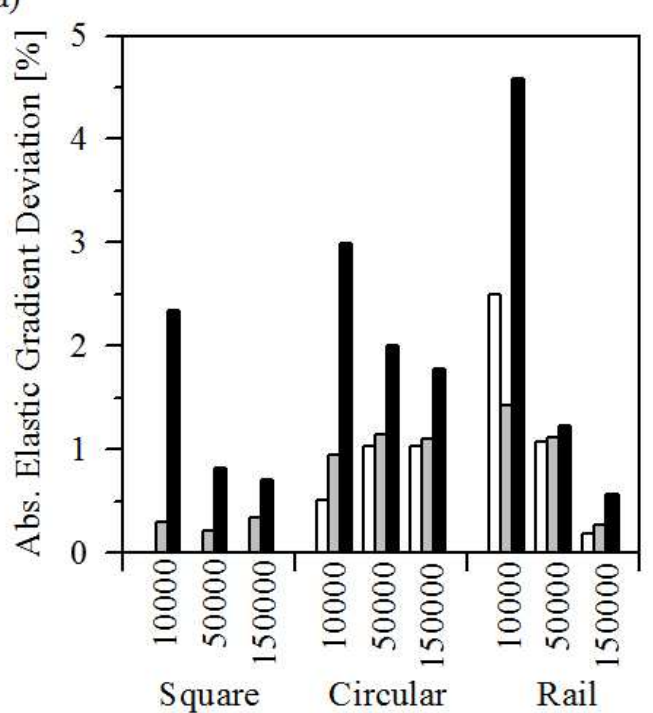

c)

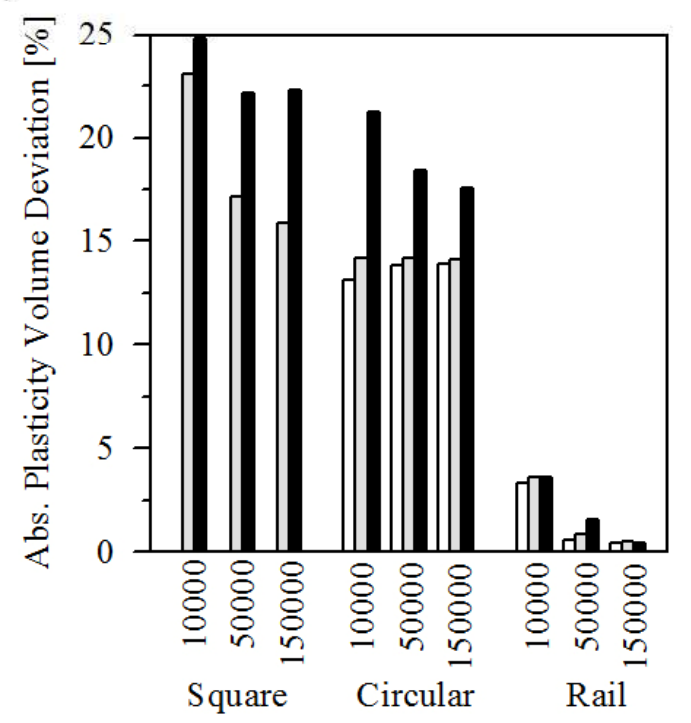

b)

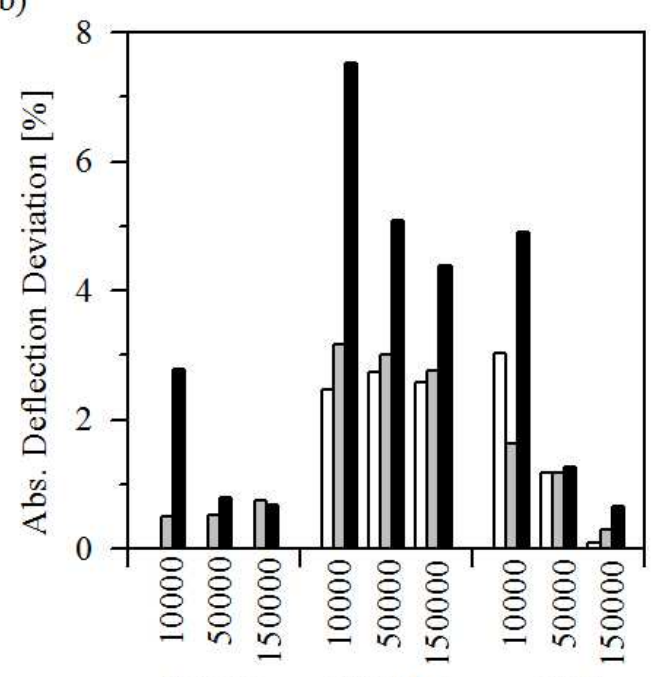

d)

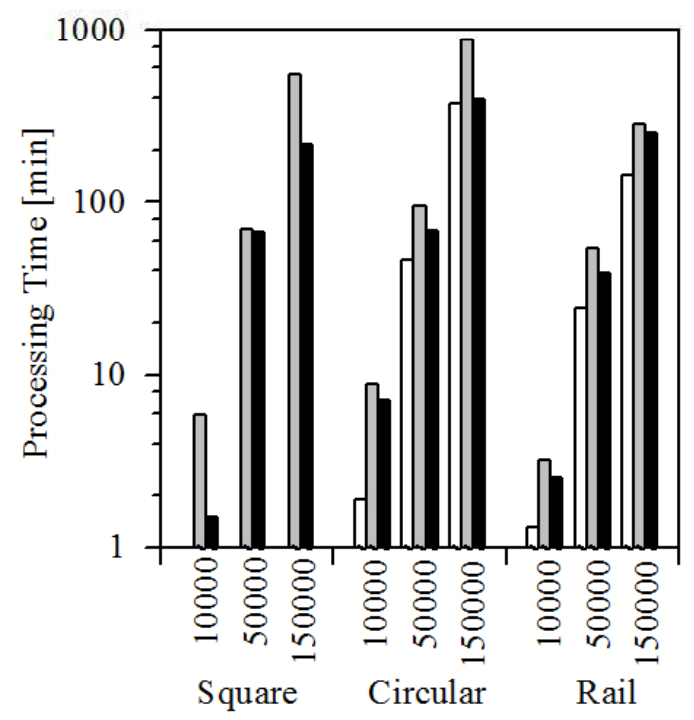

Mixed

Hex. (8)

Tet. (4)

Fig. 6. Results of the mesh comparison study for the different bending beams: a) elastic gradient; b) deflection; c) plasticity volume; d) processing time.

\subsection{Metallic Foam}

The second part of the mesh analysis simulates the compressive testing of a segment of Empore $^{\circledR}$ metal foam. In addition to the mesh types considered before, quadratic tetrahedron meshes (MSC.Marc element type 127 [9]) are investigated. No analytical solution is available for this complex geometry and therefore only a convergence analysis can be performed. In order to study the convergence behaviour, meshes with different numbers of nodes (5 000, 20 000, 50 000, 100 000, 200000 and 250000$)$ are generated. For each mesh, volume deviation, Young's modulus, the $0.2 \%$ offset yield strength and processing time are determined. The solution of the highest resolution 
mesh with 250000 nodes (hexahedron-only type) is chosen as the reference solution ( $E$ $=209.15 \mathrm{MPa}, R_{\mathrm{p} 0.2}=0.84 \mathrm{MPa}$ ). It should be mentioned here that the foam segment does not necessarily comprise a representative volume, and accordingly the obtained results cannot be interpreted as representing the macroscopic properties of Empore ${ }^{\circledR}$ aluminium foam. However, the foam segment exhibits a complex geometry and is therefore highly suitable for the comparison of finite element mesh types. The size of the meshed volume is limited due to the work-intensive meshing procedure using the software Hexpress ${ }^{\mathrm{TM}}$.

The absolute volume deviation (cf. Table 2a) describes the difference between the volume obtained by the computed tomography data and the various FE volume meshes. The value is an indicator for the accuracy of the geometric representation. An excellent geometric representation is obtained for the mixed meshes and linear tetrahedron meshes. Linear hexahedron meshes show a slightly higher deviation followed by quadratic tetrahedron and voxel meshes. The poor performance of the quadratic hexahedron meshes is due to a smaller number of elements for a fixed number of nodes. As an example, approximately 100000 nodes correspond to 66000 elements in the quadratic tetrahedron mesh and 530000 elements in the linear tetrahedron mesh. The results for Young's modulus are shown in Table $2 b$. The absolute deviation with the numerical convergence solution is given as a percentage. It can be observed that the linear hexahedron meshes and the mixed mesh converge faster towards the reference solution than the tetrahedral elements. The deviation continuously decreases with increasing numbers of elements. Interestingly, mixed type meshes already show good convergence even for a relatively low number of nodes (50 000 nodes). In comparison, a strong deviation is obtained by the quadratic tetrahedral elements which might be caused by the poor geometric representation of the volume (cf. Table 2a) due to a lower number of elements. Very poor results are obtained for the voxel type meshes with a deviation of over $70 \%$.

The stress-strain response of metal foam generally does not show a well recognizable yield point. Instead, the $0.2 \%$ offset yield strength is determined. To this end, a line with the gradient $E$ that intersects the $x$-axis at value $0.2 \%$ is drawn. The stress at the intersection of this line and the stress-strain curve is reported as the $0.2 \%$ offset yield strength. This parameter (cf. Table 2c) shows the same trend already observed for Young's modulus: linear hexahedron and mixed meshes show a faster convergence than linear tetrahedrons meshes. Again, the quadratic tetrahedron and voxel meshes yield poor results.

The processing time (computational weight) is considered in Table 2d. Linear hexahedron and voxel meshes require high processing times for calculation. In comparison, the processing of tetrahedron and mixed meshes is faster. For 100000 nodes, the quadratic tetrahedron mesh requires the lowest processing time. 


\begin{tabular}{|c|c|c|c|c|c|c|}
\hline \multicolumn{7}{|c|}{ a) Abs. Volume Deviation [\%] } \\
\hline Nodes & 250000 & 200000 & 100000 & 50000 & 20000 & 5000 \\
\hline Hex. (8) & 1.44 & 1.71 & 3.01 & N/A & N/A & N/A \\
\hline Mixed & $\mathrm{N} / \mathrm{A}$ & 0.60 & 1.00 & 1.60 & 3.25 & 9.92 \\
\hline Tet. (4) & N/A & 0.69 & 1.11 & 2.43 & 3.23 & N/A \\
\hline Tet. (10) & N/A & N/A & 4.42 & N/A & N/A & $\mathrm{N} / \mathrm{A}$ \\
\hline Voxel & $\mathrm{N} / \mathrm{A}$ & 31.95 & 36.89 & N/A & N/A & N/A \\
\hline \multicolumn{7}{|c|}{ Reference is obtained from the CT-record $=699134.94 \mathrm{~mm}^{3}$} \\
\hline Nodes & 250000 & 200000 & 100000 & 50000 & 20000 & 5000 \\
\hline Hex. (8) & Reference* & 0.03 & 7.75 & N/A & N/A & N/A \\
\hline Mixed & N/A & 1.27 & 1.43 & 1.33 & 1.97 & 10.25 \\
\hline Tet. (4) & N/A & 4.42 & 5.67 & 6.87 & 10.10 & N/A \\
\hline Tet. (10) & N/A & N/A & 54.28 & N/A & N/A & N/A \\
\hline Voxel & N/A & 72.69 & 88.77 & N/A & N/A & N/A \\
\hline \multicolumn{7}{|c|}{$*$ Reference $=209.15 \mathrm{MPa}$} \\
\hline \multicolumn{7}{|c|}{ c) Abs. $\mathbf{R}_{\mathrm{p} \mathrm{0.2}}$ Deviation [\%] } \\
\hline Nodes & 250000 & 200000 & 100000 & 50000 & 20000 & 5000 \\
\hline Hex. (8) & Reference* & 0.01 & 4.42 & N/A & N/A & N/A \\
\hline Mixed & N/A & 0.89 & 1.74 & 2.65 & 8.21 & 12.47 \\
\hline Tet. (4) & N/A & 5.10 & 7.20 & 13.26 & 17.42 & N/A \\
\hline Tet. (10) & N/A & N/A & 43.28 & N/A & N/A & N/A \\
\hline Voxel & N/A & 71.47 & 85.23 & N/A & N/A & N/A \\
\hline \multicolumn{7}{|c|}{$*$ Reference $=0.83 \mathrm{MPa}$} \\
\hline \multicolumn{7}{|c|}{ d) Processing Time [min] } \\
\hline Nodes & 250000 & 200000 & 100000 & 50000 & 20000 & 5000 \\
\hline Hex. (8) & 2820 & 1390 & 960 & N/A & N/A & N/A \\
\hline Mixed & N/A & 1340 & 530 & 420 & 90 & 10 \\
\hline Tet. (4) & N/A & 1100 & 530 & 85 & 50 & N/A \\
\hline Tet. (10) & N/A & N/A & 390 & N/A & N/A & N/A \\
\hline Voxel & N/A & 1500 & 1420 & N/A & N/A & N/A \\
\hline \multicolumn{7}{|c|}{ e) Distorted Elements [\%] } \\
\hline Nodes & 250000 & 200000 & 100000 & 50000 & 20000 & 5000 \\
\hline Hex. (8) & 51.16 & 53.72 & 61.02 & N/A & N/A & N/A \\
\hline Mixed & N/A & 60.91 & 69.68 & 76.91 & 82.13 & 94.76 \\
\hline Tet. (4) & $\mathrm{N} / \mathrm{A}$ & 93.03 & 95.23 & 96.64 & 97.45 & N/A \\
\hline Tet. (10) & $\mathrm{N} / \mathrm{A}$ & N/A & 97.67 & N/A & N/A & N/A \\
\hline Voxel & N/A & 0 & 0 & N/A & N/A & N/A \\
\hline
\end{tabular}

Table 2: Results for the Empore ${ }^{\circledR}$ foam. 


\subsection{Discussion}

A comparative analysis of the results shows a clear trend: mixed and hexahedron meshes are superior to voxel and tetrahedron meshes. This is in good agreement with earlier investigations [4-7]. In the following, the performance of mixed and hexahedron meshes will be compared in detail.

First, let us consider the bending beams. The mesh generation for the relatively simple beam geometries is unproblematic for both mesh types. The elastic solution of the bending beams is benchmarked using the elastic gradient of the force-deflection curve (cf. Fig. 4a). For the circular and rail cross section, mixed meshes showed a faster convergence towards the reference solution. It should be mentioned here that in the case of the square cross-section, no mixed mesh was created since this would simply have resulted in another hexahedron mesh. These findings are confirmed for non-linear material behaviour (deflection Fig. 6b and plasticity volume Fig. 6c). Again, mixed meshes show a slightly better performance than hexahedron-only meshes. Evaluation of processing times shows faster processing of mixed meshes in comparison with hexahedron meshes.

The superior performance of mixed meshes is confirmed in the analysis of the metallic foam segment. A hexahedron mesh with 250000 nodes is chosen as a reference solution for the convergence analysis. It is important to note that this reference solution exhibits a volume deviation of $1.44 \%$ to the target geometry. Accordingly, small deviations in the results are likely to be caused by this geometric deviation. Mixed meshes allow an accurate geometric representation of the metallic foam segment and showed the lowest volume deviation among all mesh types (cf. Table $2 \mathrm{a}$ ). The generation of onlyhexahedron meshes imposes a major constraint on the geometric complexity. The meshing procedure is extremely work-intensive in comparison with the generation of mixed meshes. In addition, the maximum size of hexahedron meshes is currently restricted by the ability of the meshing software to address sufficient amount of RAM. The elastic solution (Young's modulus, cf. Table 2b) shows fast convergence for mixed meshes. Hexahedron meshes only yield good convergence for more than 100000 nodes. A similar behaviour is observed for $0.2 \%$ offset yield strength (cf. Table 2c). Finally, mixed meshes require lower processing time than hexahedron ones (cf. Table $2 \mathrm{~d}$ ). One likely explanation for the superior performance of mixed meshes is their lower number of distorted elements. The additional use of tetrahedral- and pentahedral elements in mixed meshes allows for an accurate approximation of complex shapes without the necessity of strongly distorted element that may arise in hexahedron-only meshes or tetrahedron meshes. This theory is supported by the results displayed in Figure 7 and for the foam segment in Table 2e. Elements with a distortion value greater than the user specified threshold value are identified as distorted. The analysis is performed with a threshold value of 0.3 . The distortion of an element is calculated by evaluating the 
angles between the edges of the element. It can clearly be seen that mixed meshes have less distorted elements than hexahedron meshes.

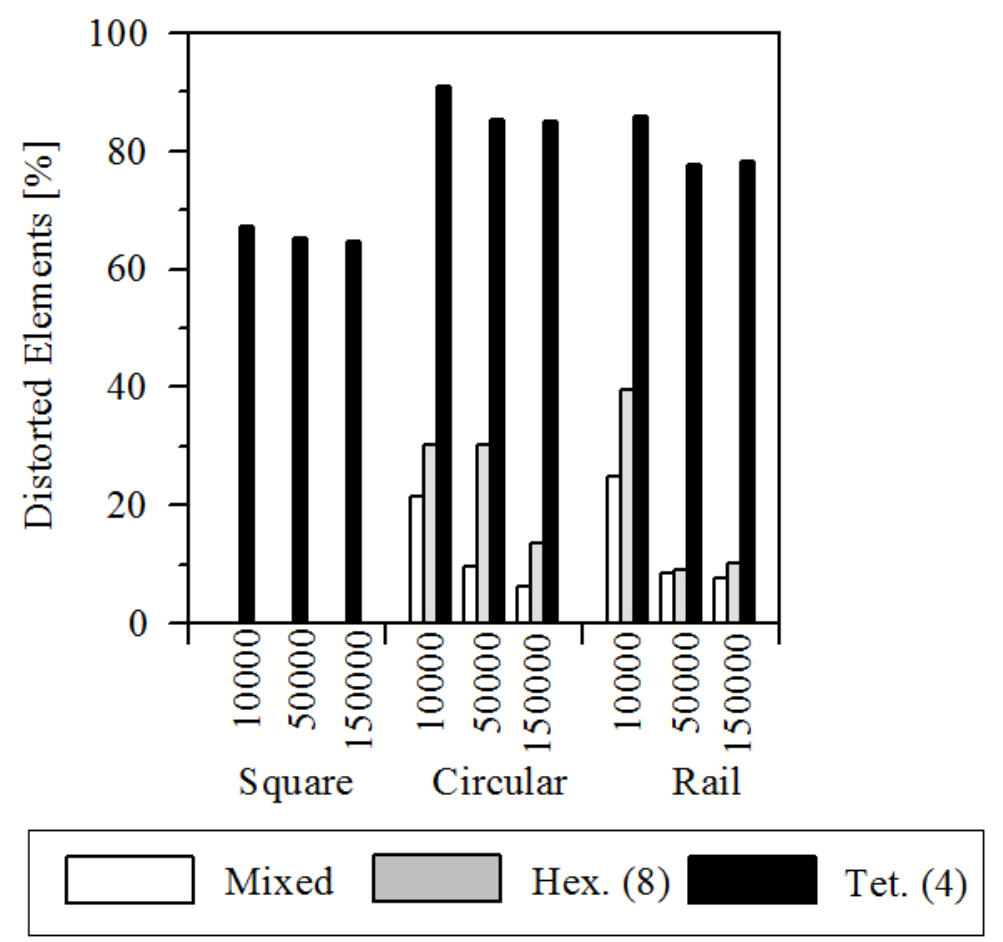

Fig. 7. Distorted elements in the beam geometries.

\section{Conclusion}

In the present study, bending beams with different cross-sections and the compression of a metal foam segment were simulated. Where possible, results were compared with analytical benchmark solutions. In all other cases, numerical convergence analysis was performed. The results indicate that linear mixed meshes are superior to linear hexahedron, linear- and quadratic tetrahedron and linear voxel meshes. Mixed meshes yield accurate results and allow a simple mesh generation. In addition, they are characterised by an accurate volume representation and fast processing times. With respect to the calculation accuracy, linear hexahedron meshes achieve comparable results. However, unlike mixed meshes, the meshing of complex geometries is workintensive and currently restricted to smaller models. In addition, hexahedron models require slightly longer processing times. Tetrahedron and voxel meshes showed mostly poor results, in particular with respect to the accuracy of results, and should be avoided for non-linear mechanical finite element analysis. 


\section{Acknowledgement}

Christoph Veyhl gratefully acknowledges the University of Newcastle, Centre for Mass and Thermal Transport in Engineering Materials for its funding support. The authors would like to thank the University of Applied Sciences Aalen, Germany for the preparation of the micro-computed tomography records. 


\section{References}

[1] M. F. Ashby, A. Evans, N. A. Fleck, Metal Foams: A Design Guide, Butterworth Heinemann, 2000.[2] R. Huiskes, E. Y. S. Chao, A survey of finite element analysis in orthopedic biomechanics: The first decade, Journal of Biomechanics 16 (1983) 385409.

[3] A. Verma, R. J. Melosh, Numerical tests for assessing finite element model convergence, International Journal for Numerical Methods in Engineering 24 (1987) 843-857.

[4] E. Wang, T. Nelson, R. Rauch, Back to Elements - Tetrahedra vs. Hexahedra, 2004 International ANSYS Conference Proceedings (2004).

[5] A. O. Cifuentes, A. Kalbag, A performance study of tetrahedral and hexahedral elements in 3-D finite element structural analysis, Finite Elements in Analysis and Design 12 (1992) 313-318.

[6] S. E. Benzley, E. Perry, K. Merkley, B. Clark, G. Sjaardema, A Comparison of AllHexahedral and All-Tetrahedral Finite Element Meshes for Elastic and Elasto-Plastic Analysis, Proceedings, 4th International Meshing Roundtable, Sandia National Laboratories (1995) 179-191.

[7] M. Viceconti, L. Bellingeri, L. Cristofolini, A. Toni, A comparative study on different methods of automatic mesh generation of human femurs, Medical Engineering \& Physics 20 (1998) 1-10.

[8] A. Ramos, J. A. Simões, Tetrahedral versus hexahedral finite elements in numerical modelling of the proximal femur, Medical Engineering \& Physics 28 (2006) 916-924.

[9] MSC.Marc, User Documentation Marc® 2007 r1 Volume B: Element Library, in: 2007.

[10] M. H. Hoskins, R. F. Kunz, J. E. Bistline, C. Dong, Coupled flow-structurebiochemistry simulations of dynamic systems of blood cells using an adaptive surface tracking method, Journal of Fluids and Structures In Press, Corrected Proof (2009). [11] K. Kovalev, Unstructured Hexahedral Non-conformal Mesh Generation, in: Faculty of Engineering, Vrije Universiteit Brussel, 2005.

[12] ASM International, ASM Handbook: Properties and Selection : Nonferrous Alloys and Special-Purpose Materials, 1990.

[13] D. Gross, W. Hauger, J. Schröder, W. A. Wall, Technische Mechanik, Band 1-4, Springer Verlag, 2008.

[14] B. Stok, M. Halilovic, Analytical solutions in elasto-plastic bending of beams with rectangular cross section, Applied Mathematical Modelling 33 (2009) 1749-1760.

[15] V. Matthews, Bahnbau, Teubner, 2003. 